\title{
Induction of the Neutrophil Migration in Normal Subjects due to Asthmatic Bronchoalveolar Lavage Fluid (BALF)
}

\author{
Ji-Sook Lee ${ }^{1, \dagger}$, Eugene Choi ${ }^{2}$, Eun Ju Yang ${ }^{3}$, Na Rae Lee ${ }^{4}$, Seung Yeop Baek, \\ Eun Jeong Kim ${ }^{5}$ and In Sik Kim ${ }^{4,5, \uparrow}$ \\ ${ }^{I}$ Department of Clinical Laboratory Science, Wonkwang Health Science University, Iksan 570-750, Korea \\ ${ }^{2}$ Department of Respiratory Internal Medicine, College of Medicine, Konyang University, Daejeon 302-718, Korea \\ ${ }^{3}$ Department of Clinical Laboratory Science, Daegu Haany University, Gyeongsangbuk-do 712-715, Korea \\ ${ }^{4}$ Department of Biomedical Laboratory Science, School of Medicine, Eulji University, Daejeon 301-746, Korea \\ ${ }^{5}$ Department of Senior Healthcare, BK21 Plus Program, Graduated School, Eulji University, Daejeon 301-746, Korea

\begin{abstract}
Human neutrophils play an essential role in the innate immune response and are involved in the pathogenesis of the
\end{abstract} \\ severe and corticosteroid-resistant asthma. Asthma is characterized by an infiltration of inflammatory cells into the lung \\ and by a cytokine release. The aim of this study is to investigate the effects of a bronchoalveolar lavage fluid (BALF) \\ on the chemotaxis and apoptosis of neutrophils which were isolated from healthy subjects. The BALF of subjects with \\ asthma induces the blood neutrophil chemotaxis in the opposite of that in normal subjects. The IL-8, IL-6, and monocyte \\ chemoattractant protein-1 (MCP-1) levels in BALF were higher in subjects with asthma than in normal subjects. The \\ BALF of normal and asthmatic subjects has no effect on neutrophil apoptosis of BALF. MCP-1 delays the constitutive \\ apoptosis of normal blood neutrophils, but has no effect in normal BALF neutrophils. These results may indicate that \\ inflammatory factors secreted by the lung tissue of patients with asthma trigger the neutrophil chemotaxis and also induce \\ the neutrophil dysregulation.
}

Key Words: Neutrophils, Asthma, Bronchoalveolar lavage fluid, Chemotaxis

\section{INTRODUCTION}

Human neutrophils have a very short half-life time of $6 \sim 18$ hours during the blood circulation and 1 4 days in tissues as a result of their constitutive apoptosis regulation by exterior ligands (Savill et al., 1989). Neutrophils act as

\footnotetext{
*Received: March 9, 2014 / Accepted: March 24, 2014

${ }^{\dagger}$ Corresponding author: In Sik Kim. Department of Biomedical Laboratory Science, School of Medicine, Eulji University 143-5, Yeuongdu-dong, Jung-gu, Daejeon 301-746, Korea.

Tel: +82-42-259-1753, Fax: +82-42-259-1759 e-mail: orientree@eulji.ac.kr

${ }^{\dagger}$ Corresponding author: Ji-Sook Lee. Department of Clinical Laboratory Science, Wonkwang Health Science University 501, Iksandaero, Iksan 570-750, Korea.

Tel: +82-63-840-1216, Fax:+82-63-840-1219

e-mail: jslee1216@wu.ac.kr

(C) The Korean Society for Biomedical Laboratory Sciences. All rights reserved.
}

key cells in the innate immune response and are also involved in airway inflammations including asthma, chronic bronchitis and chronic obstructive pulmonary diseases (COPD) (Lacoste et al., 1993; Witko-Sarsat et al., 2000). Neutrophilic inflammation has been reported in patients suffering from severe asthma (Fahy et al., 1995; Jatakanon et al., 1999).

Asthma is an allergic disease that is characterized by airway obstruction, bronchial hyperresponsiveness and mucus hypersecretion (Fireman, 2003). Although airway eosinophilic inflammation is recognized as an important feature of stable asthma, severe and corticosteroid-resistant patients with asthma show increased neutrophil counts in their airways (Douwes et al., 2002). Neutrophils can be attracted to the lungs from the blood circulation via the action of various chemoattractants. The persistent accumu- 
lation of neutrophils by a delayed constitutive apoptosis leads to the release of cytotoxic molecules, resulting in pathogenic lesions (Schenkel et al., 1982; Simon, 2003). Cytokine and chemokine regulate a variety of pathophysiological responses, including leukocyte recruitment, cell differentiation and cell survival in the inflammatory response (Murdoch and Finn, 2000). Although the role of $\mathrm{CC}$ chemokine in eosinophils and monocytes has been known well in asthma, the function of $\mathrm{CC}$ chemokine in neutrophils is still controversial (Baggiolini et al., 1997; Kim et al., 2013). Our previous report has demonstrated that the bronchoalveolar lavage fluid (BALF) of subject with asthma induces the neutrophils chemotaxis isolated from the peripheral blood of those subjects (Choi et al., 2011). Based on these results, we investigated the effects of BALF on the normal neutrophil chemotaxis and apoptosis and the association of this effect with the cytokine and chemokine in BALF. In addition, we examined the difference between blood neutrophils and BALF neutrophils by an evaluation of the alteration of neutrophil apoptosis due to the monocyte chemoattractant protein-1 (MCP-1) named as $\mathrm{CC}$ chemokine ligand 2 (CCL2).

\section{MATERIALS AND METHODS}

\section{Reagents}

RPMI 1640, antibiotics, fetal bovine serum (FBS) and Trizol were purchased from Life Technologies, Inc. (Gaithersburg, MD). Recombinant human MCP-1 was obtained from R\&D Systems (Minneapolis, MN). An annexin V-fluorescein isothiocyanate (FITC) apoptosis detection kit was purchased from BD biosciences (San Diego, CA).

\section{Subjects}

The normal subjects had a normal lung function, no asthma history and no asthma medication was required. The patients with asthma were recruited from the Konyang University Hospital and had mild to severe disease symptoms., The symptoms were measured based on the GINA criteria (Global Initiative for Asthma) (URL: http:// www.ginasthma.org 2009). The study was approved by the
Institutional Review Board of Eulji University for normal subjects and by the Institutional Review Board of Konyang University for patients with asthma. All participants in this study gave written informed consent before the study.

\section{BALF collection}

BALF was performed by instillating $100 \mathrm{ml}$ sterile isotonic saline (five aliquots of $20 \mathrm{ml}$ ) in segments of the right lower lung lobe and sequentially suctioned. The first aliquot was discharged. During bronchoscopy, $\mathrm{FIO}_{2}$ was kept at $100 \%$. BALF was filtered through sterile gauze filters, collected on ice, and immediately centrifuged at 1,000 $\mathrm{g}$ for 20 minutes. The supernatant was stored at $-70^{\circ} \mathrm{C}$ for subsequent methods.

\section{Neutrophil isolation and cell culture}

The human neutrophils were isolated from the heparinized peripheral blood and BALF of normal subjects using the Ficoll-Hypaque gradient centrifugation and a CD 16 microbeads magnetic cell sorting kit (Miltenyi Biotec, Bergisch Gladbach, Germany). The erythrocytes were removed using the RBC lysing solution (Sigma Korea, Seoul. Korea). After that the cells were re-suspended at $3 \times 10^{6}$ cells $/ \mathrm{ml}$ in an RPMI 1640 medium with $1 \%$ antibiotics and $10 \%$ FBS.

\section{Chemotaxis assay}

The neutrophil migration was evaluated by using a 48-well microchamber (Neutoprobe, Gaithersburg, MD). The lower wells were filled with $28 \mu$ l buffer alone or with BALF of normal and asthma.

A polyvinylpyrrolidone (PVP)-free filter (Neuroprobe) with $5 \mu \mathrm{m}$ sized pores was placed over the lower wells. $50 \mu \mathrm{l}$ of neutrophils at $2 \times 10^{6}$ cells $/ \mathrm{ml}$ were with the cell suspension addedinto the upper well. The assembled chamber was incubated for $90 \mathrm{~min}$ at $37^{\circ} \mathrm{C}$ in a humidified incubator. The non-migrated cells adhering to the upper surface of polycarbonate filter were removed. Then the filter was dried, fixed and stained with Diff-Quick (Baxter Diagnostics, McGaw Park, IL). The cells of four randomly selected fields per well were counted and the chemotactic index $(\mathrm{CI})$ was calculated from the number of cells which 
migrated to the control.

\section{Enzyme linked immunosorbent assay (ELISA)}

The protein concentrations in BALF were measured in the normal subjects and in the patients with asthma with a sandwich ELISA using the OptEIA ${ }^{\mathrm{TM}}$ set human MCP-1, MIP-1 $\alpha$, RANTES, TARC, IL-6, IL-8, GM-CSF, and TNF- $\alpha$ (BD bioscience) according to the manufacturer's instructions.

\section{Apoptosis assay}

For the apoptosis detection the neutrophils were incubated with the FITC-labeled annexin V and propidium iodide (PI) for $15 \mathrm{~min}$ at room temperature. The apoptotic neutrophils were analyzed using FACSCalibur with CellQuest software (BD bioscience) and were defined as the cells that stained positive for annexin $\mathrm{V}$ with or without PI. Ten thousand events were collected for each experiment.

\section{Statistical analysis}

All data are expressed as the means \pm SD. Data were analyzed by student $t$-test using the SPSS statistical software package, Version 10,0 (SPSS, Inc., Chicago, IL). A $p$-value $<0.05$ was considered significant.

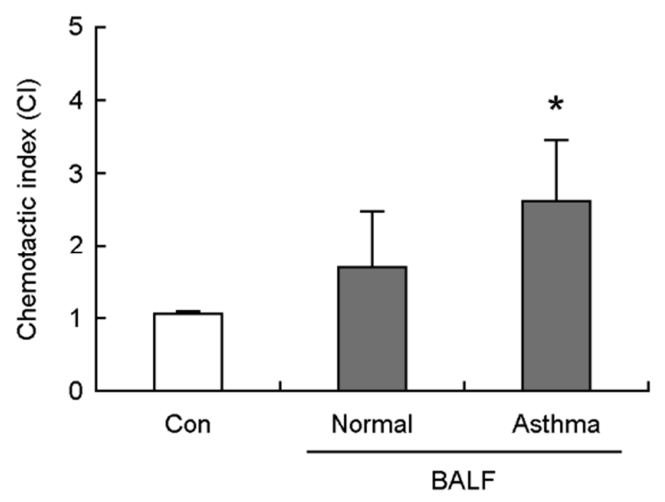

Fig. 1. Chemotaxis of blood neutrophils in response to asthmatic BALF. Neutrophils were isolated from the peripheral blood of normal subjects $(n=3)$. The cells were applied to the absence (Con) and presence of BALF of normal subjects (Normal) and patients with asthma (Asthma) in a 48-well microchamber and allowed to migrate for $90 \mathrm{~min}$. The chemotactic index (CI) was calculated from the number of cells that migrated to the control. Data are expressed as the means \pm SD. ${ }^{*} P<0.05$ indicates a significant difference between the control group and BALFtreated groups.

\section{RESULTS}

BALF of asthmatic subject induces the chemotaxis of blood neutrophils of normal subjects

The circulating neutrophils in the peripheral blood are in asthma recruited to the lung tissue inflammation sites like the lung tissue via the pro-inflammatory molecules action (Ordonez et al., 2000). These molecules are released from the lung tissue of patients with asthma (Jatakanon et al, 1999). The blood neutrophils chemotaxis was significantly induced by BALF of patients with asthma $(P<0.05)$, compared to that of normal subjects (Fig. 1). This result indicates that inflammatory factors secreted by the lung tissue of patients with asthma trigger the blood neutrophil chemotaxis.

A

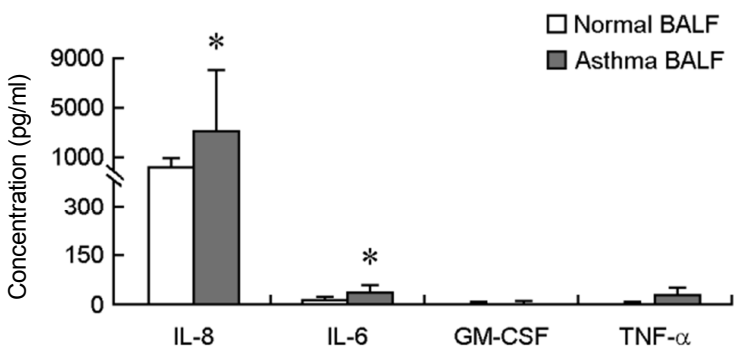

B

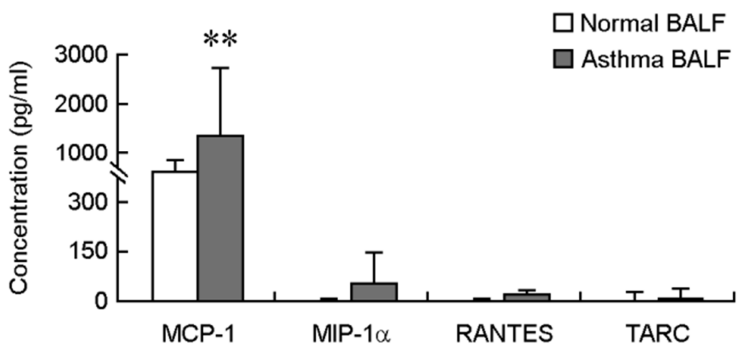

Fig. 2. The levels of cytokines and chemokines in BALF of normal and asthmatic subjects. BALF was collected from normal $(n=9)$ and asthmatic patients $(n=39)$. (A) Cytokines including IL-6, IL-8, GM-CSF, and TNF- $\alpha$ were measured by ELISA. (B) Chemokines including MCP-1, MIP-1 $\alpha$, RANTES, and TARC were measured by ELISA. Data are expressed as the means $\pm \mathrm{SD} .{ }^{*} P<0.05$ and $* * P<0.01$ indicate a significant difference between the normal BALF group and asthmatic BALF group. 


\section{IL-8, IL-6, and MCP-1 of asthmatic BALF are different} from those of normal BALF

To investigate the chemotaxis-related inflammatory factors in asthmatic BALF, both cytokines and chemokines in BALF of normal subjects and patients with asthma were analyzed using the ELISA test. As shown in Fig. 2A, the levels of IL-8 and IL-6 in BALF were significantly higher in patients with asthma than in normal subjects $(P<0.05)$. The level of MCP-1 n BALF was significantly higher in patients with asthma than in normal subjects $(P<0.01)$ (Fig. $2 \mathrm{~B})$. These results indicate that neutrophil chemotaxis in

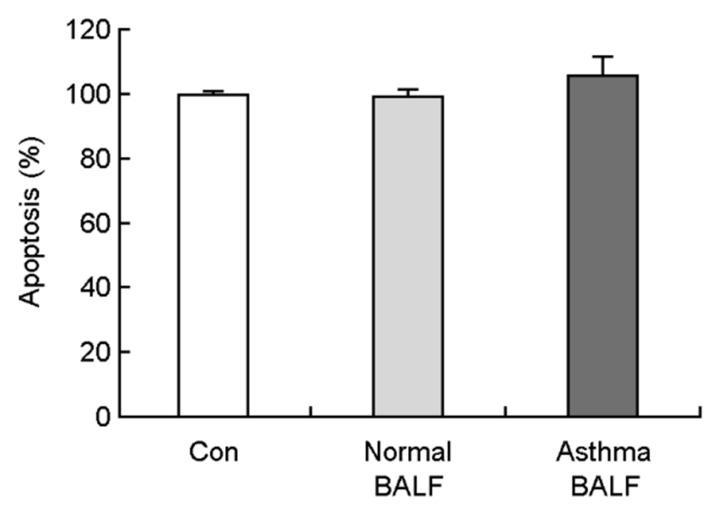

Fig. 3. The effect of normal and asthmatic BALF on apoptosis of BALF neutrophils. Neutrophils were isolated from BALF of normal subjects $(n=3)$ and were incubated with BALF of normal and asthmatic subjects for $24 \mathrm{~h}$. Data are expressed in relation to the control (Con), which was set at $100 \%$. Data are expressed as the means $\pm \mathrm{SD}$.

\section{A}

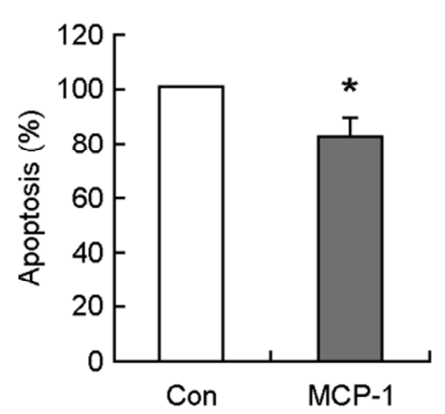

response to asthmatic BALF may be associated with increased levels of IL-8, IL-6, and MCP-1 as compared to normal BALF.

\section{The effect of normal and asthmatic BALF on apoptosis of BALF neutrophils}

IL-8, IL-6, and MCP-1 act as survival factors or antiapoptotic factors in neutrophils (Simon, 2003; Yang et al., 2012). Because IL-8, IL-6, and MCP-1 increased in BALF of patients with asthma compared to the BALF of normal subjects, we examined whether the BALF of normal subjects and patients with asthma affects the neutrophil apoptosis. As shown in Fig. 3, the BALF neutrophils were not altered by the BALF of normal subjects and patients with asthma.

The different anti-apoptotic effect of MCP-1 on the neutrophils apoptosis of the peripheral blood and BALF

Due to the fact that environmental factors such as BALF can alter the regulation of lung neutrophils, the neutrophil apoptosis of blood and BALF was examined due to an evaluation of the MCP-1 anti-apoptotic effect. MCP-1 delayed significantly the constitutive neutrophil apoptosis of the normal peripheral blood $(P<0.05)$ (Fig. 4A) However, MCP-1 had no effect on the neutrophil apoptosis of normal BALF (Fig. 4B). Thrse results indicate that blood neutrophils are different from lung neutrophils and MCP-1 has differential effects on the apoptosis of blood and BALF.

B

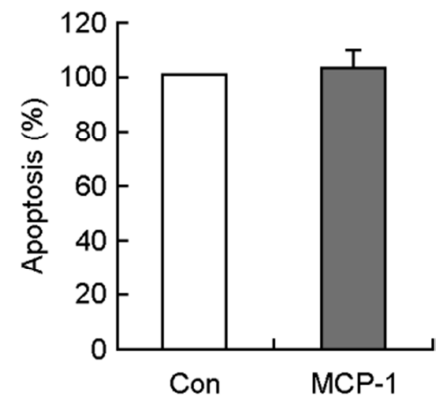

Fig. 4. Different anti-apoptotic effect of MCP-1 on neutrophil apoptosis of the peripheral blood and BALF. Neutrophils were isolated from the peripheral blood ( $n=3)(A)$ and BALF of normal subjects $(n=3)(B)$. Neutrophils were incubated for $24 \mathrm{~h}$ in the absence (Con) and presence of MCP-1 $(100 \mathrm{ng} / \mathrm{ml})$. Apoptosis was analyzed by measuring the binding of annexin V-FITC and PI. Data are presented in relation to the control, which was set at $100 \%$. Data are expressed as the means $\pm \mathrm{SD}$. ${ }^{*} P<0.05$ indicates a significant difference between the control and the MCP-1-treated groups. 


\section{DISCUSSION}

The neutrophilic inflammation is one of the important processes in the pathogenesis of asthma. In previous studies it was reported that increased sputum neutrophils are involved in an irreversible lung function loss in patients with asthma (Fahy et al., 1995). However, the mechanism of the neutrophilic inflammation in the asthmatic airway has not been clearly elucidated yet. The main results of this study are the BALF of patients with asthma induces the normal neutrophils migration, which leads to a neutrophils recruitment at the local inflammation site such as lung tissues. The increase of MCP-1, IL-6 and IL- 8 in the asthmatic BALF may be suggested as essential chemokines and cytokines in the chemotactic effect of asthmatic the BALF.

In the immune response of normal subjects, the neutrophils migrate in the peripheral blood to the inflamed site will not return to the circulation thereafter. Neutrophils are either eliminated by mucosa secretion or die in the tissues. One occasion by which neutrophils are destroyed is the apoptosis or the genetically programmed cell suicide. After apoptosis, the dead neutrophils are eliminated by phagocytes. However, the recruited neutrophils in tissues are not properly removed by these inflammatory disease processes which lead to an increased tissue damage and an inflammation resolution failure (Cross et al., 2006). In our study, the BALF of normal subjects and patients with asthma had no effect on the spontaneous apoptosis of neutrophils isolated from BALF (Fig. 3). However, the BALF collected from the lungs of patients with asthma induced the blood neutrophil chemotaxis (Fig. 1). Our hypothesis was that the cytokine alteration in the lung is associated with the chemotactic effect of BALF on neutrophils. Due to this hypothesis we investigated the concentration of cytokine or chemokine involved in the chemotaxis and activation of neutrophils. As shown in Fig. 2, the asthmatic BALF showed the increased IL-8, IL-6, and MCP-1 compared to the normal BALF. Because IL-8, IL-6 and MCP-1 function as essential molecules in the survival, migration and activation of neutrophils, the chemotactic effect induced by BALF may be involved in the direct or indirect effect of IL-8, IL-6 and
MCP-1 (Murphy et al., 2000; Zhang and Wenzel, 2007). The exact mechanism of the asthmatic BALF remains to be elucidated and is the subject of an ongoing study.

For an examination of the BALF alteration of lung neutrophils the anti-apoptotic effect of MCP-1 was evaluated in the blood and BALF neutrophils of normal subjects. It was proved in a previous study that the blood neutrophils apotposis was delayed by MCP-1 (Yang et al., 2012). On the other hand, we confirmed in the present study that MCP-1 is not effective on the apoptosis of BALF neutrophils (Fig. 4). Despite the difference between BALF and blood neutrophils further studies are necessary to examine the alteration of apoptosis of asthmatic BALF neutrophils by MCP-1.

In conclusion, our study results may suggest that a neutrophil recruitment into the lung is caused by environmental factors such as BALF which is secreted by lung structural cells.

\section{Acknowledgement}

This paper was supported by Wonkwang Health Science University in 2014.

\section{REFERENCES}

Baggiolini M, Dewald B, Moser B. Human chemokines: an update. Annu Rev Immunol. 1997. 15: 675-705.

Choi E, Yang EJ, Kim DH, Lee JS, Kim IS. CCR expression of bronchoalveolar lavage fluid (BALF) neutrophils and chemotactic activity of BALF. J Exp Biomed Sci. 2011. 17: 89-93.

Douwes J, Gibson P, Pekkanen J, Pearce N. Non-eosinophilic asthna: Importance and possible mechanisms. Thorax. 2002. 57: 643-648.

Fahy JV, Kim W, Liu J, Boushey HA. Prominent neutrophilic inflammation in sputum from subjects with asthma exacerbation. J Allergy Clin Immunol. 1995. 95: 843-852.

Fireman P. Understanding asthma pathophysiology. Allergy and Asthma Proceedings. 2003. 24: 79-83.

Jatakanon A, Uasuf C, Maziak W, Lim S, Chung KF, Barnes PJ. Neutrophilic inflammation in severe persistent asthma. Am J Respir Crit Care Med. 1999. 160: 1532-1539.

Kim IS, Kim EH, Kim DH, Kim JS, Lee JS. Effect of house dust mite and CCL2 on S100A8 and S100A9 expression in human 
monocytes. Biomed Sci Lett. 19: 344-377.

Lacoste JY, Bousquet J, Chanez P, Van Vyve T, Simony-Lafontaine J, Lequeu N, Vic P, Enander I, Godard P, Michel FB. Eosinophilic and neutrophilic inflammation in asthma, chronic bronchitis, and chronic obstructive pulmonary disease. J Allergy Clin Immunol. 1993. 92: 537-548.

Murdoch C, Finn A. Chemokine receptors and their role in inflammation and infectious diseases. Blood. 2000. 95: 3032-3043.

Murphy PM, Baggiolini M, Charo IF, He'bert CA, Horuk R, Matsushima K, Miller LH, Oppenheim JJ, Power CA. International union of pharmacology. XXII. Nomenclature for chemokine receptors. Pharmacol Rev. 2000. 52: 145-176.

Ordonez CL, Shaughnessy TE, Matthay MA, Fahy JV. Increased neutrophil numbers and IL-8 levels in airway secretions in acute severe asthma. Am J Respir Crit Care Med. 2000. 161: 1185-1190.

Savill JS, Wyllie AH, Henson JE, Walport MJ, Henson PM, Haslett C. Macrophage phagocytosis of aging neutrophils in inflammation. Programmed cell death in the neutrophil leads to its recognition by macrophage. J Clin Invest. 1989. 83: 865 -875 .

Schenkel E, Atkins PC, Yost R, Zweiman B. Antigen-induced neutrophil chemotactic activity from sensitized lung. J Allergy Clin Immunol. 1982. 70: 321-325.

Simon HU. Neutrophil apoptosis pathways and their modifications in inflammation. Immunol Rev. 2003. 193: 101-110.

Witko-Sarsat V, Rieu P, Descamps-Latscha B, Lesavre P, Halbwachs-Mecarelli L. Neutrophils: Molecules, functions and pathophysiological aspects. Lab Invest. 2000. 80: 617-653.

Yang EJ, Choi EG, Ko JS, Kim D-H, Lee J-S, Kim IS. Differential Effect of CCL2 on Constitutive Neutrophil apoptosis between normal and asthmatic subjects. J Cell Physiol. 2012. 227: 2567-2577.

Zhang JY, Wenzel SE. Tissue and BAL based biomarkers in asthma. Immunol Allergy Clin North Am. 2007. 27: 623-632. 